\title{
Articulación de la complejidad matemática de la media aritmética
}

\section{Articulation of the mathematical complexity of the arithmetic mean}

\author{
Carlos Rondero \\ Centro de Investigación en Matemáticas. Universidad Autónoma del Estado de Hidalgo \\ rondero@uaeh.reduaeh.mx \\ Vicenç Font \\ Departament de Didàctica de les Ciències Experimentals i de la Matemàtica. Universitat de Barcelona \\ vfont@ub.edu
}

RESUMEN • Las reflexiones sobre la complejidad de los objetos matemáticos, y la articulación de los componentes de esta complejidad, son frecuentes en muchos de los enfoques teóricos utilizados en el área de la Educación Matemática. En este artículo, se realiza una reflexión teórica, en el marco del Enfoque ontosemiótico de la cognición e instrucción matemática, que profundiza en los mecanismos de articulación de la complejidad asociada al objeto matemático. Se presenta una visión integrada de los diferentes tipos de articulación contemplados en trabajos de investigación previos, en los que se ha utilizando como marco teórico este enfoque: niveles de generalización, proyecciones metafóricas y tramas de funciones semióticas. Para ello, como contexto de reflexión, se utiliza el objeto matemático «media aritmética».

PALABRAS CLAVE: complejidad del objeto matemático; articulación; media aritmética; enfoque ontosemiótico.

ABSTRACT • Reflections on the complexity of the mathematical objects and the necessary articulation of the components of such complexity are common in many of the theoretical approaches used in the area of Mathematics Education. In this paper we present a theoretical reflection, inside the framework of the Onto-Semiotic Approach of the Cognition and Mathematical Instruction, that explores the mechanisms of articulation of the complexity associated to the mathematical object. We present an integrated view of different types of articulation covered in previous research works that have been using this approach as a theoretical framework (layers of generality, metaphoric projection and sequence of semiotic functions. With this purpose, we use the mathematical object «arithmetic mean» as a context of reflection.

KEYWORDS: complexity of the mathematical object; articulation; arithmetic mean; onto-semiotic approach. 


\section{INTRODUCCIÓN}

Una característica de la Educación Matemática es la amplia diversidad de perspectivas teóricas. Cada una de ellas tiende a privilegiar alguna de las dimensiones del proceso de enseńanza-aprendizaje de las Matemáticas sobre las demás; pero hay un aspecto en el que muchas de estas teorías coinciden, nos referimos a que consideran que una característica de los objetos matemáticos que deben ser enseńados y aprendidos es su complejidad. Precisamente, en nuestra opinión, la complejidad de los objetos matemáticos -conjuntamente con el hecho de que solo a través de representaciones semióticas es posible una actividad sobre ellos (D’Amore, Fandińo, Iori y Matteuzzi, 2015)-, y la complejidad de su proceso de enseñanza-aprendizaje son unas de las razones de que exista una pluralidad de teorías en el área de Educación Matemática, y que en estos momentos se plantee la necesidad del dialogo y la articulación de teorías (Prediger, Bikner-Ahsbahs y Arzarello, 2008; Radford, 2008a).

La complejidad del objeto matemático lleva a pensar no en un objeto unitario sino en un sistema complejo formado por partes o componentes. Nos encontramos pues con que un objeto matemático, cuando participa en la actividad matemática, queda inmerso en un juego de lenguaje (Wittgenstein, 1953) en el que se habla del objeto desde la dualidad unitaria-sistémica. En algunas circunstancias, los objetos matemáticos participan como entidades unitarias (que se supone que son conocidas previamente), mientras que en otras intervienen como sistemas que se deben descomponer para su estudio. Por ejemplo, en el estudio de la adición y sustracción en los últimos niveles de educación primaria, el sistema de numeración decimal (decenas, centenas, etcétera) se considera como algo conocido y, en consecuencia, como entidades unitarias (elementales). Este mismo objeto, en el primer curso de primaria, tiene que ser considerado de manera sistémica para su aprendizaje. Un elemento esencial para que sea posible esta mirada dual hacia los objetos matemáticos es que los componentes que resultan de la mirada compleja se articulan (conectan) entre sí, posibilitando la mirada unitaria del objeto matemático.

Las reflexiones sobre la complejidad y la articulación son frecuentes en muchos de los enfoques teóricos utilizados en el área de la Educación Matemática. En el caso de la Teoría antropológica de lo didáctico (Chevallard, 1992), la mirada compleja sobre los objetos matemáticos que ofrece esta teoría lleva a pensar no en un objeto simple sino en una praxeología, o más exactamente un conjunto de praxeologías que se articulan como resultado de un proceso de modelización. La descripción de la dinámica y complejidad de los objetos y las prácticas matemáticas (y didácticas), en términos de la articulación de praxeologías matemáticas (y didácticas) de complejidad creciente, ha sido específicamente descrita en Barquero, Bosch y Gascón (2011 y 2013).

En el caso de la Teoría APOE (acción, proceso, objeto, esquema), los objetos emergen como resultado de dos mecanismos: encapsulación y tematización. La encapsulación es un mecanismo basado en la abstracción reflexiva. Se refiere a la posibilidad de pensar en un proceso como algo completado, y ser capaz de caracterizarlo y estudiar sus propiedades. A través de la encapsulación, las nociones abstractas se conciben como objetos con propiedades y varias representaciones. La tematización implica la posibilidad de pensar el esquema como un todo para actuar o hacer transformaciones sobre él y estudiar sus propiedades. También se involucra la posibilidad de diseccionar, analizar, examinar sus partes y volver a componerlo como un todo. La tematización va de la complejidad (formada por varios esquemas) a la unidad del objeto reificado. En el APOE, (Badillo, 2003; Baker, Cooley y Trigueros, 2000) se considera la articulación en términos de coordinación de esquemas que se desarrollan según la tríada intra, inter y trans, propuesta por Piaget y García (1982). En este enfoque, se considera que la coordinación de esquemas juega un papel relevante en el proceso de tematización.

En la Teoría de la objetivación (Radford, 2008b), los objetos matemáticos, conceptos, signos y significado están entrelazados a través de la actividad reflexiva generada para resolver un conjunto de 
problemas, que son cultural y socialmente significativos. En la teoría de la objetivación, la mirada compleja lleva a considerar que el objeto conceptual no es un objeto monolítico u homogéneo, es un objeto conformado de capas de generalidad. Cada capa está asociada con una actividad reflexiva particular, determinada por las características de los significados semióticos de la objetivación que median. En esta teoría, el proceso de generalización es uno de los principales mecanismos de articulación.

En la Ciencia cognitiva de las matemáticas (Lakoff y Núñez, 2000), se considera que las estructuras matemáticas que las personas construyen tienen su origen en los procesos cognitivos relacionados con la vida diaria, tales como esquemas de imágenes y el pensamiento metafórico. Lakoff y Núnez (2000) afirman que las metáforas crean una relación conceptual entre un dominio de partida y un dominio de llegada, y distinguen dos tipos de metáforas conceptuales que tienen relación con las matemáticas: las metáforas conectadas a tierra (grounding), que relacionan un dominio de partida fuera de las Matemáticas con un dominio de llegada en el interior de las Matemáticas, y las metáforas de conexión (linking), que tienen en la misma matemática tanto el dominio de partida como el dominio de llegada. En esta teoría la dualidad unitaria-sistémica tiene un papel central. Por una parte, la metáfora es unitaria (A es B). Por otra parte, la metáfora nos permite generar un nuevo sistema de prácticas (perspectiva sistémica) como resultado de nuestra comprensión del dominio de llegada en términos del dominio de partida. Lakoff y Núnez (2000) desarrollan la dualidad unitaria-sistémica de diferentes metáforas. Para ello, dada una metáfora, los dominios de partida y de llegada se descomponen mediante un proceso de análisis para determinar los conceptos, las propiedades, relaciones, etcétera que son transferidas desde el dominio de salida al de llegada. En esta teoría, el proceso de articulación se conceptualiza básicamente en términos de proyecciones metafóricas.

En mayor o menor medida, la problemática de la complejidad asociada al objeto matemático, y la articulación de los componentes en los que estalla esta complejidad, está presente en casi todos los marcos teóricos emergentes en el área de la Educación Matemática. En este artículo tomaremos como referente teórico el Enfoque ontosemiótico de la cognición e instrucción matemática (EOS, a partir de ahora) (Godino, Batanero y Font, 2007).

En el marco del EOS, se ha profundizado sobre la mirada sistémica de los objetos matemáticos, y se ha introducido la idea de significados parciales y la descripción de estos significados parciales en términos de prácticas y configuraciones de objetos primarios, activados en estas prácticas. Esta mirada compleja se ha aplicado a diferentes objetos matemáticos. Por ejemplo, en Godino, Font, Wilhelmi y Arrieche (2009), para el caso del número natural; en Wilhelmi, Godino y Lacasta (2007), para la noción de igualdad, y en Pino, Godino y Font (2011) para la derivada. En cambio, sobre la noción de articulación se ha profundizado menos. En diferentes trabajos, en los que se usa el EOS como marco teórico, la articulación entre los componentes de una configuración epistémica se modela por medio de funciones semióticas. Por ejemplo, en Godino, Font, Wilhelmi y Lurduy (2011) y en Rojas (2012, 2013 y 2015). La articulación entre configuraciones se ha modelado en términos de, por un lado, generalización (o mayor formalismo), por ejemplo en Godino, Font, Wilhelmi y Arrieche (2009), Wilhelmi, Godino y Lacasta (2007) y Pino, Godino y Font (2011), y por otro, proyecciones metafóricas, por ejemplo en Acevedo (2008).

El objetivo de este artículo es realizar una reflexión teórica, en el marco del EOS, cuyo objetivo es profundizar en la articulación de la complejidad asociada al objeto matemático como un paso previo y necesario para pasar a una visión unitaria del objeto matemático. Se pretende presentar una visión integrada de los diferentes tipos de articulación contemplados en trabajos de investigación previos, en los que se ha utilizado como marco teórico el EOS. Para ello, como contexto de reflexión se utiliza el objeto «media aritmética». Hemos seleccionado este objeto porque, a diferencia de otros como la derivada o la integral que normalmente son considerados complejos, suele ser considerado más bien como un objeto simple. Ahora bien, en nuestra opinión la media aritmética tiene una característica 
de transversalidad en diferentes campos de la Matemática, por lo que resulta un buen contexto para reflexionar acerca de la complejidad y la articulación.

El camino que seguiremos será el siguiente: en primer lugar, mostraremos la complejidad asociada al objeto de media aritmética; en segundo lugar, comentaremos diferentes maneras de relacionar, conectar o articular (en nuestra terminología) los diferentes componentes de esta complejidad, y por último, reflexionaremos sobre cómo esta articulación permite pasar de una visión compleja sobre la media aritmética a una visión unitaria sobre este objeto.

\section{COMPLEJIDAD DE LA MEDIA ARITMÉTICA}

Diferentes investigaciones realizadas sobre la media aritmética (Callingham, 1997; Cobo y Batanero, 2004; Leavy y O’Loughlin, 2006; García Cruz y Garrett, 2008; Ortiz y Font, 2014) han puesto de manifiesto que cuando se utiliza la media aritmética en las prácticas matemáticas, además de su definición, se usan diferentes representaciones, determinadas características y propiedades, otros objetos matemáticos relacionados como son las otras medias, etcétera. Una buena herramienta para mostrar esta complejidad son los mapas conceptuales. A continuación (figura 1), sigue un mapa conceptual elaborado por los autores que muestra algunos contextos intramatemáticos en los que se realizan prácticas matemáticas en las que intervienen definiciones, propiedades, representaciones, etcétera relacionadas con la media, con el objetivo de mostrar una parte de la complejidad asociada a la media aritmética.

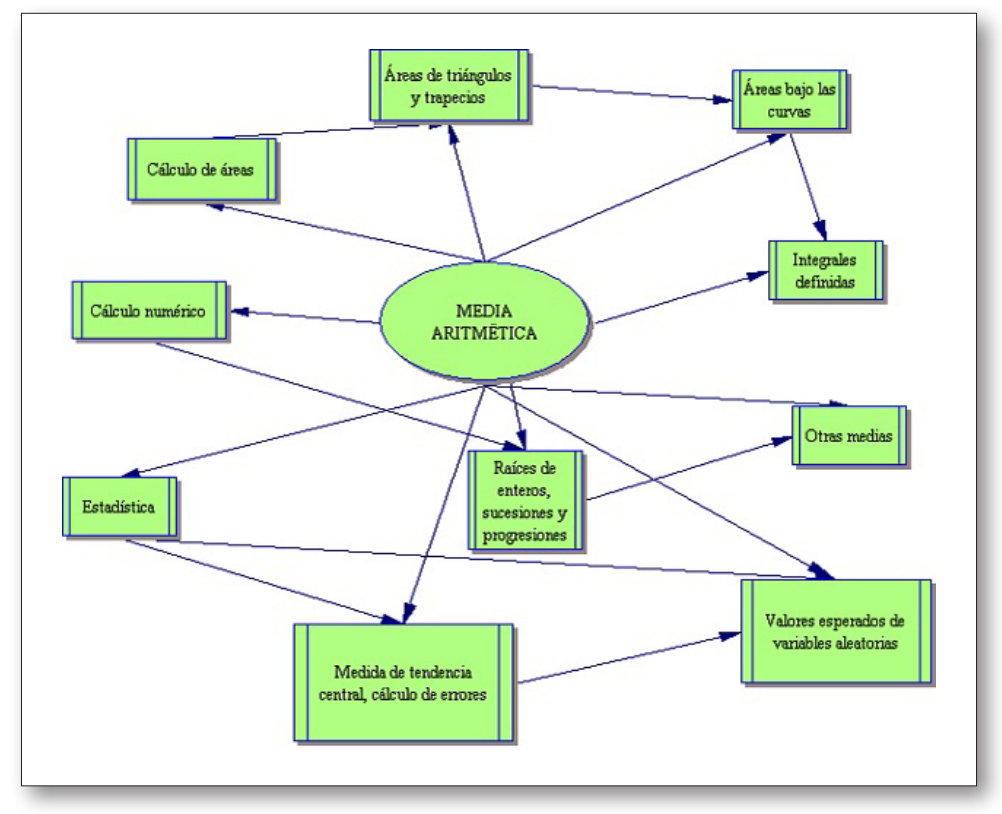

Fig. 1. Mapa conceptual de la media aritmética.

Otro instrumento útil para describir la complejidad (sin buscar la exhaustividad) del conglomerado de representaciones, definiciones, propiedades, tipos de problemas, etcétera que a lo largo del tiempo se han ido sucediendo para el estudio de la media aritmética, es la herramienta de configuración epistémica. Según Font, Godino y Gallardo (2013), para analizar un texto matemático y, más en general, la actividad matemática, sea profesional o escolar, es necesario contemplar como mínimo los siguientes elementos: en primer lugar, notaciones, representaciones (lenguaje): en segundo lugar, situacionesproblema; en tercer lugar, definiciones; en cuarto lugar, procedimientos, técnicas, etcétera; en quinto 
lugar, proposiciones, propiedades, teoremas, etcétera, y por último, argumentos. Estos seis tipos de elementos se articulan formando configuraciones epistémicas. Se trata de una herramienta que puede ser útil para describir la complejidad de los objetos matemáticos y de las prácticas de las que emergen.

En el EOS, la introducción de la dualidad unitaria-sistémica permite reformular la visión «ingenua» de que "hay un mismo objeto matemático (media aritmética) con distintas representaciones». Lo que hay es un sistema complejo de prácticas que permiten resolver problemas, en las que el objeto matemático «media aritmética» no aparece directamente, lo que sí aparece son representaciones de la media aritmética, diferentes definiciones de la media aritmética, proposiciones y propiedades de esta, procedimientos y técnicas que se aplican a la media aritmética y argumentos sobre ella. Dicho de otra manera, a lo largo de la historia se han ido generando diferentes configuraciones epistémicas para el estudio de la media aritmética, algunas de las cuales han servido para generalizar las preexistentes.

La noción de media aritmética se ha usado, a lo largo de la historia de las matemáticas, en diversas prácticas matemáticas: por un lado, el método babilónico y el de Herón de Alejandría para calcular raíces de enteros positivos; por otro, el método de Arquímedes para el equilibrio de los cuerpos, y el cálculo de áreas y volúmenes, el método de Merton para estudiar el movimiento uniformemente acelerado, etcétera. Este conjunto de prácticas se puede parcelar en diferentes subconjuntos de prácticas que se realizan gracias a la activación de determinadas configuraciones epistémicas, algunas de las cuales se pueden considerar como reorganizaciones y generalizaciones de las anteriores.

Hemos podido identificar para la media aritmética diferentes contextos intramatemáticos y extramatemáticos, a cada uno de los cuales se les asocia un conjunto de prácticas matemáticas y una configuración epistémica que las permite realizar. A estas configuraciones las denominamos como sigue: en primer lugar, el método babilónico y de Herón para calcular raíces, (CE1); en segundo lugar, el método de Arquímedes sobre el equilibrio de los pesos, principio básico de la estática (CE2); en tercer lugar, sucesiones, progresiones aritméticas y geométricas (CE3); en cuarto lugar, método de Merton para el estudio del movimiento (CE4), y por último, cálculo de áreas, métodos de cuadraturas, fórmulas, etcétera (CE5).

La media aritmética, a lo largo de su evolución histórica, se ha activado implícita o explícitamente en al menos estos cinco subsistemas de prácticas, cada uno de los cuales tiene una configuración epistémica asociada. Estas configuraciones, a pesar de ser distintas entre sí, presentan articulaciones entre ellas, de manera que se pueden relacionar como se ilustra en el esquema de la figura 3, atendiendo al mayor grado de generalidad.

En la base del esquema de la figura 3, se han considerado cinco «sistemas de prácticas» y las configuraciones activadas en estos sistemas (CE1, CE2, CE3, CE4 y CE5); en ellas se resuelven ciertos tipos de situaciones-problemas con métodos y procedimientos particulares, etcétera. Sin embargo, dentro de estas cinco configuraciones hay algunas que son similares entre sí. Tal es el caso de las configuraciones CE1, CE2, CE3, y CE5 que se pueden agrupar dando origen a un sistema de prácticas más «genérico» en el cual se abordan situaciones-problemas sobre cálculo numérico. A este sistema de prácticas lo denominamos numérico. Análogamente, las configuraciones CE2, CE4 y CE5 se pueden agrupar dando paso a un nuevo sistema de prácticas más general que denominamos áreas, ya que se abordan problemas de este tipo.

En un siguiente nivel, se presenta la configuración de un sistema de prácticas más genérico que da lugar a la configuración CE6, a la que llamamos estadistica, y a la configuración CE7, cálculo de integrales definidas. De todas estas configuraciones, emerge una referencia global llamada media aritmética.

Cuando se hace intervenir la dualidad unitaria-sistémica, las relaciones de articulación por medio de la generalización dan cuenta de la complejidad de la media aritmética y de la multiplicidad de sus espacios de intervención dentro de la Matemática. La participación es diversa, la configuración epistémica CE1 se refiere a que en la Antigüedad los babilonios y los griegos la usaron para el cálculo de 
raíces de enteros positivos (uno de los métodos más conocidos es el de Herón de Alejandría). Por otra parte, uno de los libros más importantes de la Matemática china (Chiu Chang Suan Shu), contiene un capítulo que se titula precisamente «Exceso y Defecto» (Needham, 1992), dedicado a la invención en el Álgebra china de la regla de la falsa posición, usada, sobre todo, para resolver ecuaciones del tipo $\mathrm{ax}=\mathrm{b}$. Precisamente, la regla de la falsa posición es usada, entre otros, por Herón de Alejandría en su método de obtención de raíces cuadradas de enteros positivos en el que interviene el exceso y el defecto (y, posteriormente, también en el método de Newton para el cálculo de raíces de funciones). La configuración epistémica CE2 se refiere a la manera en que Arquímedes ocupa el exceso y el defecto, como elemento epistemológico subyacente en el estudio del equilibrio, lo que a su vez le permite calcular áreas y volúmenes de diferentes figuras geométricas y centros de gravedad de algunas de ellas.

En el caso de la configuración epistémica CE3, en una referencia particular al caso de las progresiones aritméticas, la intervención de la media aritmética se da sobre todo en el cálculo de las sumas, dado que las sumas de $n$ términos de estas son equivalentes a la media aritmética del primer y el último término, en cada caso.

La configuración epistémica CE4 se refiere a la llamada Ley de Merton. La figura 2, construida para explicar el movimiento, es un trapecio cuya área representa el espacio recorrido por el cuerpo, y hace intervenir a la velocidad media, calculada a su vez como la media aritmética de la velocidad inicial y la velocidad final. En este caso, el exceso está representado por el triángulo que se forma por encima de la velocidad media y el defecto por el triángulo por debajo de la velocidad media.

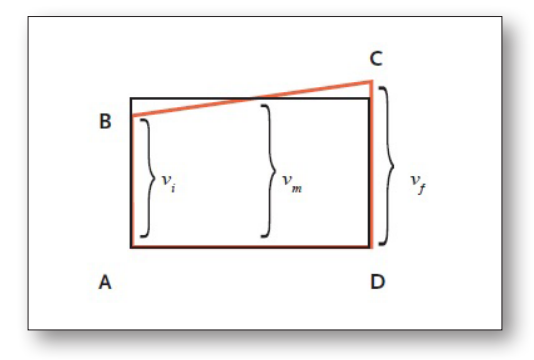

Fig. 2. Ley de Merton.

Finalmente, en el caso de la configuración epistémica CE5, referida al cálculo de áreas, intervienen varios métodos de cuadraturas (Cavallieri, Fermat, Pascal, Wallis, etcétera) para encontrar el área bajo la curva, en algunos de los cuales se hace intervenir de una u otra forma a la media aritmética aunque, en ocasiones, esto no es del todo explicitado por los propios autores.

En la figura 3 se muestra, por una parte, la complejidad asociada a la media aritmética y, por otra parte, la articulación de las configuraciones epistémicas de la media aritmética mediante niveles de generalización. 


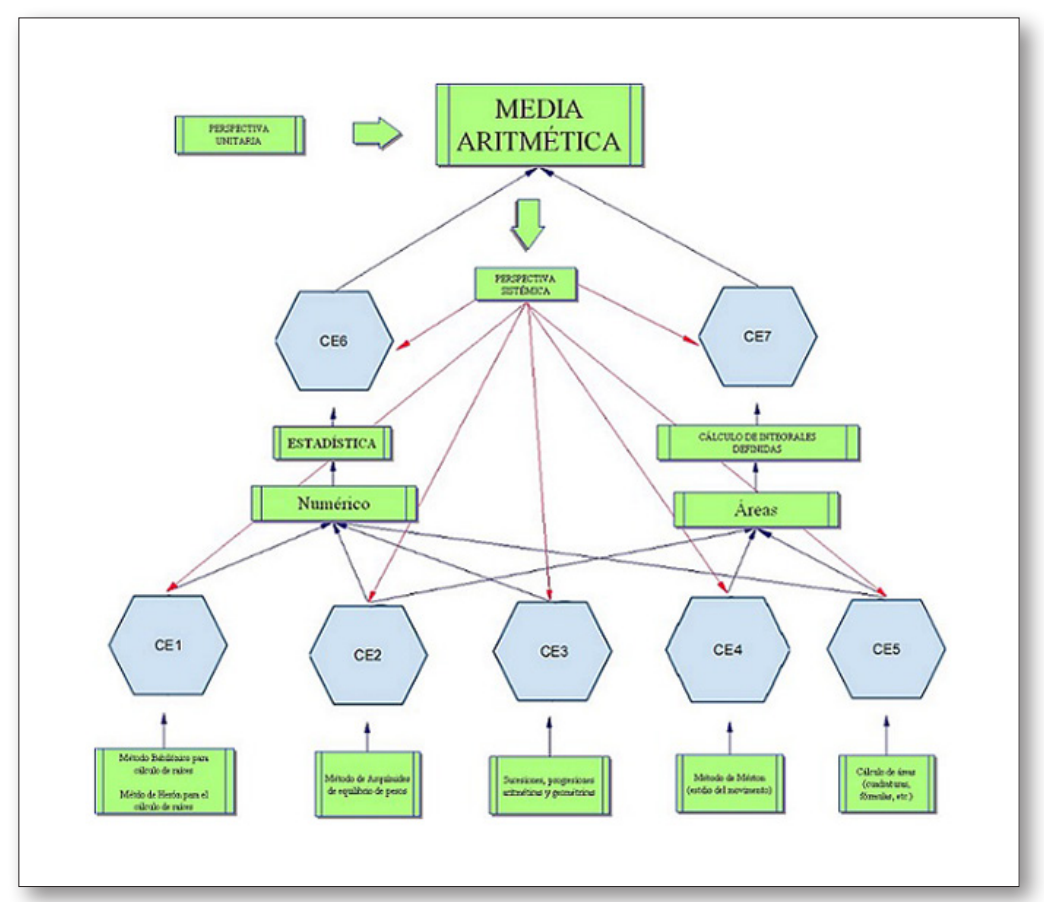

Fig. 3. La dualidad unitaria-sistémica en el caso de la media aritmética.

A continuación se desarrolla, en el cuadro 1, la CE6 que hemos llamado «CE Estadística», porque en ella aparece lo que se considera como paradigma de la definición de media aritmética $\left(\bar{x}=\frac{\sum x_{i}}{n}\right)$. Esta configuración se puede considerar como una generalización y reorganización de las configuraciones CE1 y CE2 que, en nuestra opinión, son las que llevan a conceptualizar la media como $\bar{x}=\frac{\sum x_{i}}{n}$.

El objetivo del desarrollo de la CE6, que se presenta en el cuadro 1, es mostrar parte del complejo de representaciones, definiciones, proposiciones, propiedades, etcétera asociadas al término media aritmética que aparecen cuando este se usa en el contexto intramatemático de la estadística. En la primera fila, se ha seleccionado una muestra representativa de problemas en cuya resolución interviene de manera determinante la media aritmética; en la segunda fila, una muestra de representaciones ostensivas; en la tercera, las diferentes definiciones; en la cuarta, las propiedades más relevantes, y en la quinta, los argumentos que se utilizan para resolver los problemas seleccionados en la primera fila. Por cuestiones de espacio no se dan detalladas las definiciones (fila 3) de esta configuración y tampoco se desarrollan las otras configuraciones. 
Cuadro 1.

Configuración Epistémica «Estadística»

\section{Problemas}

En esta configuración se resuelven problemas como los siguientes (Batanero, 2000):

Problemas en los que hay que buscar un valor que compensa los excesos con los defectos (equilibrio, equidad, etcétera):

Ejemplo P1. Unos nińos llevan a clase caramelos. Andrés lleva 5, María 8, José 6, Carmen 1 y Daniel no lleva ninguno. ¿Cómo repartir los caramelos de forma equitativa?

Problemas en los que hay que hacer la estimación de una medida:

Ejemplo P2. Ocho alumnos de la misma clase miden el peso de un objeto pequeño usando el mismo instrumento, obteniendo los siguientes valores en gramos: 6,2;6,0;6,0;6,3;6,1;6,23;6,15; 6,2. ¿Cuál sería la mejor estimación del peso real del objeto?

Problemas en los que se comparan dos conjuntos de valores dados utilizando la media como valor representativo de cada conjunto de datos:

Ejemplo P3. Los siguientes valores se obtuvieron al medir la altura $(\mathrm{cm})$ alcanzada al saltar por un grupo de alumnos antes y después del entrenamiento. ¿Crees que el entrenamiento es efectivo?

Altura alcanzada en cm

\begin{tabular}{|l|c|c|c|c|c|c|c|c|c|c|}
\hline Alumnos & Ana & Bea & Carol & Diana & Elena & Fanny & Laia & Hilda & Inés & Juana \\
\hline Antes del entrenamiento & 115 & 112 & 107 & 119 & 115 & 138 & 126 & 105 & 104 & 115 \\
\hline Después del entrenamiento & 128 & 115 & 106 & 128 & 122 & 145 & 132 & 109 & 102 & 117 \\
\hline
\end{tabular}

Problemas en los que, dado un conjunto de datos no todos conocidos y su media aritmética, hay que buscar el dato desconocido:

Ejemplo P4. La altura media de los alumnos de un colegio es 1'40. Si extraemos una muestra aleatoria de 5 estudiantes y resulta que la altura de los 4 primeros es de 1’38, 1’42, 1’60, 1’40. ¿Cuál sería la altura más probable del quinto estudiante?

Problemas en los que está involucrado un promedio de promedios:

Ejemplo P5. Hay 10 personas en un ascensor, 4 mujeres y 6 hombres. El peso medio de las mujeres es de 60 kilos y el de los hombres de 80 . ¿Cuál es el peso medio de las 10 personas del ascensor?

\section{Lenguaje}

Verbal:

Relacionados con el contexto (por ejemplo, en el problema P3, entrenamiento, salto, etcétera).

Términos verbales matemáticos (suma, división, media aritmética, etcétera).

Simbólico:

Números enteros, decimales y fracciones. Símbolos de suma, producto y división, expresiones simbólicas

como $\bar{x}=\frac{\sum x_{i}}{n}=\frac{x_{1}+x_{2}+\ldots+x_{n}}{n}, P=\frac{x_{1} m_{1}+x_{2} m_{2}+\ldots+x_{n} m_{n}}{m_{1}+m_{2}+\ldots+m_{n}}, P=\frac{\sum_{i=1}^{n} x_{i} m_{i}}{\sum_{i=1}^{n} m_{i}}$, etc.
Tabular:

Tablas (por ejemplo, como en el problema P3)

Gráfico-simbólico

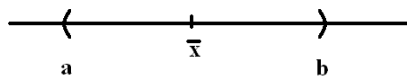




\section{Definiciones-conceptos}

Exceso, defecto, suma, multiplicación, división, medida, estimación, ponderación (cada valor $\mathrm{x}_{\mathrm{i}}$, tiene asignado una ponderación $\mathrm{m}_{\mathrm{i}}$ ),

media aritmética (suma de todos los valores dividida por el número de valores)

media aritmética (estimación de una medida de una magnitud)

media aritmética (valor representativo de un conjunto de datos)

media aritmética (operador que asocia a un conjunto de datos un único valor)

promedio de promedios

media ponderada

Conceptos estadísticos que pueden estar relacionados con la media aritmética (frecuencia, marca de clase en un histograma, rango, mediana, varianza, desviación estándar, coeficiente de variación, centro o punto medio de una distribución bidimensional, covarianza de dos variables de una distribución bidimensional, coeficiente de correlación, recta de regresión, valor esperado de una variable aleatoria discreta o continua, desviación estándar de una variable aleatoria discreta o continua, etcétera).

\section{Proposiciones}

Dados dos números positivos a y $\mathrm{b}$, con $\mathrm{a}<\mathrm{b}$, la media aritmética de estos dos números está a la misma distancia de a que de $b$.

Los excesos y los defectos se compensan:

$$
P=\left(P-x_{1}\right) m_{1}+\left(P-x_{2}\right) m_{2}+\ldots+\left(P-x_{n}\right) m_{n}=0, P=\sum_{i=1}^{n}\left(P-x_{i}\right) m_{i}=0
$$

$\bar{x} .\left(\mathrm{n}^{\mathrm{o}}\right.$ de valores $)=$ total

La mejor estimación del peso real es la media.

Proposiciones de Estadística y Probabilidad en las que interviene la media, como por ejemplo:

Si la distribución de frecuencias es unimodal y bastante simétrica, se cumple aproximadamente que: a) en el intervalo $(\bar{x}-\sigma, \bar{x}+\sigma)$ hay un $68 \%$ de la población (s es la desviación estándar); b) en el intervalo $(\bar{x}-2 \sigma, \bar{x}+2 \sigma)$ hay un $95 \%$ de la población; c) en el intervalo $(\bar{x}-3 \sigma, \bar{x}+3 \sigma)$ hay un $99 \%$ de la población.

En una distribución normal, se cumple que el área limitada por función y los intervalos $(\mu-\sigma, \mu+\sigma)$, $(\mu-2 \sigma, \mu+2 \sigma),(\mu-3 \sigma, \mu+3 \sigma)$ es igual a $0,6826 \mathrm{u}^{2} ; 0,9544 \mathrm{u}^{2}$ y $0,9974 \mathrm{u}^{2}$ respectivamente hay un $68 \%$ de la población (s es la desviación estándar); b) en el intervalo ( $\bar{x}-2 \mathrm{~s}, \bar{x}-2 \mathrm{~s}$ ) hay un $95 \%$ de la población; c) en el intervalo $(\bar{x}-3 \mathrm{~s}, \bar{x}+3 \mathrm{~s})$ hay un $99 \%$ de la población.

\section{Procedimientos}

Sumar, dividir, multiplicar, estimación, cálculo mental.

Cálculo de la media (se suman todos los valores y después se divide por el número de valores).

Comparación y ordenación de medias.

Resolución de ecuaciones (para hallar un valor desconocido, conociendo la media y los otros datos).

Resolución de problemas (hay que hacer intervenir adecuadamente los datos dados).

Procedimientos estadísticos que pueden estar relacionados con la media aritmética (cálculo de la media aritmética de una distribución de frecuencias, cálculo de la media por calculadora, cálculo de la varianza y de la desviación estándar, cálculo de la covarianza y del coeficiente de correlación, método de los mínimos cuadrados, etcétera). 
Argumentos (por ejemplo):

Para P1. La media es el valor equitativo porque todos tienen la misma cantidad y no queda ningún caramelo para repartir.

Para P2. Al medir se cometen errores, es de esperar que estos se compensen, por tanto la media representa la medida del peso más representativa y confiable.

Para P3. (a) Se espera que el entrenamiento haya sido efectivo, eso ocurrirá si el promedio de los saltos después de este tiene un valor mayor que antes del entrenamiento; (b) la media de salto antes era 115,6 y ahora es de 120,4; y 120,4 >115,6. Conclusión, el entrenamiento es efectivo.

Para P4. La media de la muestra es de esperar que coincida con la media de la población. El valor desconocido es aquel que se halla conociendo la media $(1,40)$ y los otros datos.

Para P5. Cuando aparecen datos y otros distintos referidos a los primeros, estos se identifican como valores ponderados. En este caso cada dato es un promedio, el resultado es un promedio ponderado de promedios.

\section{ARTICULACIÓN DE LA COMPLEJIDAD EN EL CASO DE LA MEDIA ARITMÉTICA}

En este apartado consideraremos tres mecanismos de articulación de la complejidad asociada a nuestro contexto de reflexión: los niveles de generalidad, la metáfora y la articulación semiótica.

\section{Niveles de generalidad}

En la sección se ha explicado cómo en la figura 3 las configuraciones epistémicas C6 y C7 están en un nivel de generalidad superior a las C1-C4. Las configuraciones C6 y C7 son el resultado de procesos de reconstrucción y articulación de complejidad crecientes aplicados sobre $\mathrm{C} 1-\mathrm{C} 4$, cuyo resultado son configuraciones cuyo dominio de aplicación es cada vez más general. La figura 3 se relaciona, en cierta manera, con la distinción entre Matemáticas formales e informales considerada en Lakatos (1981), en particular con la progresión de las informales hacia las formales.

Al subir a capas de generalidad más elevadas, se puede perder la conexión o relación con las capas inferiores. Por ejemplo, si definimos en la CE6 el valor esperado de una variable aleatoria continua como:

$$
\text { Sea X una variable aleatoria continua. El valor esperado de X es } E(x)=\int_{-\infty}^{+\infty} x f(x) d x
$$

El alumno puede no entender que esta definición está relacionada con la idea de media aritmética, que precisamente es la razón de ser de la propia definición.

Para que se produzca la articulación entre las capas de generalidad, es necesario explicitar la relación entre ellas, ya sea mostrando claramente que hay una relación particular-general (por ejemplo, que la media aritmética es un caso particular de la media ponderada), ya sea mostrando que una configuración epistémica es el resultado de la reestructuración de otras configuraciones epistémicas, de manera que los problemas que se resuelven con esta nueva $\mathrm{CE}$ son los mismos que se resuelven con las que reorganiza, pero que a su vez resuelve otros nuevos o más generales.

A continuación comentamos otro tipo de articulaciones o conexiones que no quedan del todo englobadas en la idea de capa de abstracción, formalización generalización o modelización. Nos referimos a la metáfora y a lo que aquí llamaremos articulación semiótica. 


\section{Metáforas}

De acuerdo con Rondero (2010), consideramos que la metáfora conceptual (Lakoff y Núñez, 2000) que hay detrás de la media aritmética es la siguiente: «La media aritmética es el equilibrio entre los excesos y los defectos». Esta metáfora suele estar sugerida por expresiones metafóricas como la que se halla en el problema 1 de la configuración epistémica CE6: «... ¿ ¿ómo repartir los caramelos de forma equitativa?». Otras expresiones metafóricas que sugieren esta metáfora conceptual que hemos encontrado en libros de texto son, por ejemplo: «La media aritmética o promedio destaca por representar el reparto equitativo, el equilibrio, la equidad»; «el valor que tendrían los datos, si todos ellos fueran iguales»; «el valor que correspondería a cada uno de los datos de la distribución si su suma total se repartiera por igual», $y$ «la media aritmética puede ser denominada como centro de gravedad de una distribución, el cual no está necesariamente en la mitad», etcétera.

Consideramos que la metáfora sugerida por Rondero (2010) es una metáfora de tipo grounding en la que el dominio de partida es la configuración epistémica CE2, realizada por Arquímedes para el estudio del equilibrio (un dominio de fuera de las Matemáticas), y el dominio de llegada, en lugar de ser pesos, es parte de la configuración epistémica CE6 (valores numéricos). Esta metáfora es sugerida por algunas expresiones metafóricas (como las que acabamos de citar) que se hallan en los textos cuando se quiere dar una idea intuitiva de la media, pero no es directamente sugerida por la fórmula usada para calcular la media aritmética de dos valores a y b: $\mathrm{P}=(\mathrm{a}+\mathrm{b}) / 2$. La tabla 1 , elaborada siguiendo el modelo tabular de Lakoff y Núnez (2000), muestra la proyección metafórica de esta metáfora conceptual.

Tabla 1.

Proyección metafórica de conexión entre CE2 y CE6

\begin{tabular}{|l|l|}
\hline \multicolumn{1}{|c|}{ Dominio de partida (CE2) } & \multicolumn{1}{c|}{ Dominio de llegada (CE6) } \\
\hline Centro de gravedad & Media aritmética de dos valores a y $\mathrm{b}(\mathrm{con} \mathrm{a}>\mathrm{b})$ \\
\hline $\begin{array}{l}\text { Dos pesos } \mathrm{w}_{1} \mathrm{y}_{2} \text {, colocados a distancias } \mathrm{d}_{1} \mathrm{y} \\
\mathrm{d}_{2}, \text { del punto de equilibrio } \\
\mathrm{w}_{1} \mathrm{~d}_{1}=\mathrm{w}_{1} \mathrm{~d}_{2}\end{array}$ & $\begin{array}{l}\text { Ponderación } \\
\text { Cada valor } \mathrm{x}_{\mathrm{i}} \text {, tiene asignado una ponderación } \mathrm{p}_{\mathrm{i}}\end{array}$ \\
\hline Exceso & $\mathrm{E}>0, \mathrm{E}=\mathrm{P}-\mathrm{a}$ \\
\hline Defecto & $\mathrm{D}<0, \mathrm{D}=\mathrm{P}-\mathrm{b}$ \\
\hline Exceso diferente de defecto $\rightarrow$ Desequilibrio & $\mathrm{E}$ diferente de $-\mathrm{D} \rightarrow$ Desequilibrio \\
\hline Exceso igual a menos defecto $\rightarrow$ Equilibrio & $\mathrm{E}$ igual a $-\mathrm{D} \rightarrow \mathrm{Equilibrio}$ \\
\hline Los excesos compensan los defectos & $\mathrm{P}=\mathrm{a}+\mathrm{E}, \mathrm{P}=\mathrm{b}+\mathrm{D}, \mathrm{E}=-\mathrm{D}$ \\
& $2 \mathrm{P}=\mathrm{a}+\mathrm{b}$ \\
& $\mathrm{P}=(\mathrm{a}+\mathrm{b}) / 2$ \\
& $\left(P-x_{1}\right) m_{1}+\left(P-x_{2}\right) m_{2}+\ldots+\left(P-x_{n}\right) m_{n}=0$ \\
& $\sum_{i=1}^{n}\left(P-x_{i}\right) m_{i}=0$ \\
\hline
\end{tabular}

Ahora bien, a su vez, según nuestra opinión, el dominio de partida (estudio del equilibrio) es un dominio extramatemático que está estructurado a partir de la proyección metafórica del esquema de imagen «equilibrio» (Johnson, 1987). Este esquema de imagen emerge de nuestras experiencias de interacción física y social con nuestros cuerpos y con otras entidades en el mundo: 
... tenemos un caso de concepto universal y abstracto de equilibrio, con la fórmula «Toda X está equilibrada cuando es simétrica, o sea, donde hay elementos iguales, con pesos iguales y emplazamientos iguales, uno a cada lado con relación al eje o punto central, o cuando hay la misma distribución de peso y fuerzas respecto del eje» (Johnson, 1987: 152).

La proyección de este esquema de imagen nos permite hablar y razonar acerca de diferentes tipos de entidades abstractas como si ellas fueran el resultado del equilibrio que resulta de compensar excesos con defectos. Se trata de otra proyección metafórica de tipo grounding (conectada a tierra) que describimos en la tabla 2.

Tabla 2 .

Proyección metafórica conectada a tierra

\begin{tabular}{|c|c|}
\hline Dominio de partida (esquema de imagen equilibrio) & Dominio de llegada (CE2) \\
\hline $\begin{array}{l}\text { Experiencias corporales del tipo: andar en bicicleta, } \\
\text { caminar sin caerse, percibir la homeostasis del cuerpo } \\
\text { o hacer malabares, generan el siguiente esquema de } \\
\text { imagen: }\end{array}$ & Equilibrio de pesos \\
\hline $\begin{array}{l}\text { El control del cuerpo nos permite estar erguidos (sa- } \\
\text { bemos controlar nuestro centro de gravedad). } \\
\text { Un cuerpo queda en equilibrio cuando su soporte es el } \\
\text { centro de gravedad. }\end{array}$ & $\begin{array}{l}\text { Un cuerpo queda en equilibrio cuando su soporte es el } \\
\text { centro de gravedad. }\end{array}$ \\
\hline $\begin{array}{l}\text { Si sopeso A con una mano, al sopesar otra A en la otra } \\
\text { mano, se obtiene el equilibrio. }\end{array}$ & A equilibra a A (reflexividad). \\
\hline $\begin{array}{l}\text { Si al sopesar A y B en cada mano obtengo el equili- } \\
\text { brio, al cambiar los objetos de mano se mantendrá el } \\
\text { equilibrio. } \\
\text { Si A está en una mano y B en la otra y en equilibrio, } \\
\text { si éste se mantiene al sustituir B por C, entonces A y } \\
\text { C también se equilibran sin necesidad de sopesarlas. }\end{array}$ & $\begin{array}{l}\text { Si un peso A equilibra a otro B, entonces B equilibra } \\
\text { a A (simetría). } \\
\text { Si un peso A equilibra a otro B y éste a } C \text {, entonces } A \\
\text { equilibra a C (transitividad). }\end{array}$ \\
\hline $\begin{array}{l}\text { Con los brazos extendidos tengo que hacer más fuerza } \\
\text { con el brazo donde hay más peso. } \\
\text { Cuando se va a buscar agua con un palo con un cubo } \\
\text { en cada extremo, y no están igual de llenos, el equili- } \\
\text { brio se consigue cuando el punto de apoyo de la per- } \\
\text { sona está más cerca del cubo más lleno. } \\
\text { El exceso de peso se compensa con la distancia al pun- } \\
\text { to de apoyo. } \\
\text { El equilibrio se pierde cuando el exceso no se compen- } \\
\text { sa con el defecto. }\end{array}$ & $\begin{array}{l}\text { Dos pesos } \mathrm{w}_{1} \mathrm{y} \mathrm{w}_{2} \text {, colocados a distancias } \mathrm{d}_{1} \text { y } \mathrm{d}_{2} \text {, del } \\
\text { punto de equilibrio: } \mathrm{w}_{1} \mathrm{~d}_{1}=\mathrm{w}_{1} \mathrm{~d}_{2}\end{array}$ \\
\hline
\end{tabular}




\begin{tabular}{|l|l|}
\hline \multicolumn{1}{|c|}{ Dominio de partida (esquema de imagen equilibrio) } & \multicolumn{1}{|c|}{ Dominio de llegada (CE2) } \\
\hline $\begin{array}{l}\text { Pesos iguales, la misma fuerza en cada brazo } \\
\begin{array}{l}\text { Cuando se va buscar agua con un palo con un cubo en } \\
\text { cada extremo, si los dos cubos están igual de llenos, el } \mathrm{el} \\
\text { equilibrio se consigue cuando el punto de apoyo de la } \\
\text { persona está en la mitad del palo. }\end{array}\end{array}$ & $\begin{array}{l}\text { Pesos iguales, distancias iguales al punto de apoyo: } \mathrm{w}_{1} \\
=\mathrm{w}_{2}, \mathrm{~d}_{1}=\mathrm{d}_{2}\end{array}$ \\
\begin{tabular}{l} 
Platos de la balanza equilibrados \\
\hline
\end{tabular}
\end{tabular}

La razón por la que consideramos plausible la proyección metafórica del esquema de acción «equilibrio» en la obra de Arquímedes son los postulados que toma como principios en su libro De l'équilibre des plans ou des centres de gravité des plans. Arquímedes inicia su libro diciendo que asume como punto de partida siete afirmaciones que, en nuestra opinión, es plausible considerar que se deriven de experiencias corporales relacionadas con el equilibrio. Se trata de afirmaciones como la siguiente:

I. Nous demandons que des poids égaux s'equilibrent a`des distances égales, et que des poids égaux a’des distances inégales ne s'equilibrant pas, mais qu'il y ait inclinaison du côté des poids placé a la plus grande distance (Obras completas de Arquimedes, Tomo I, 1960: 303).

Por otro lado, en la CE6 que hemos llamado estadistica se usa una linking metáfora para articular la estadística descriptiva con la inferencial. En la estadística descriptiva, se trabaja con datos simples o agrupados, y se calcula tanto la media aritmética como la varianza, que es una medida que toma de referencia a la media para calcular una media del cuadrado de desviaciones, todo lo cual está inmerso en un modelo determinista, puesto que en la estadística descriptiva no aparece la medida de probabilidad. Ya en la estadística inferencial, tanto la media aritmética como la varianza se presentan en otro nivel de generalidad. Pero en este caso ya interviene la probabilidad ya que, dada la característica aleatoria de la muestra de la población, estamos inmersos en una modelación indeterminista (aleatoria). En la estadística inferencial, intervienen las variables aleatorias, ya sean discretas (binomial, geométrica, Poisson, etcétera) o continuas (uniforme, normal, gamma, etcétera), se hace referencia a sus correspondientes funciones de densidad de probabilidad y funciones de distribución de estas para luego proceder, en cada caso, a calcular tanto el valor esperado (media) como la varianza.

En esta CE6 «estadística» se usa la linking metáfora (de conexión) de «el valor esperado es la media aritmética» para relacionar la estadística descriptiva y la estadística inferencial. Un ejemplo de expresión metafórica de esta metáfora conceptual la podemos encontrar en el siguiente libro de texto (alumnos de secundaria de 17 ańos), cuando se introduce por primera vez la noción de esperanza matemática (valor esperado) a partir de un contexto de un juego de ganancias según la carta que se obtiene al extraer una carta de una baraja:

Este valor recibe el nombre de media aritmética de la variable aleatoria X o esperanza matemática e indica la ganancia media esperada al realizar la experiencia aleatoria un gran número de veces (Barceló, Bujosa, Cañadilla, Fargas y Font, 2002: 391).

Otro ejemplo lo hallamos en la entrada esperanza matemática de la Wikipedia:

En estadística la esperanza matemática (también llamada esperanza, valor esperado, media poblacional o media) de una variable aleatoria es el número que formaliza la idea de valor medio de un fenómeno aleatorio (<http://es.wikipedia.org/wiki/Esperanza_matem\%C3\%A1tica>).

En algunos casos, esta linking metáfora se combina con la grounding metáfora comentada anteriormente: 
El valor esperado representa el valor promedio que se espera suceda al repetir el experimento en forma independiente una gran cantidad de veces. El valor esperado se interpreta físicamente como el centro de masa o centro de gravedad de la distribución de probabilidad (...) (<http://148.204.211.134/polilibros/Portal/ Polilibros/P_terminados/PROBABILIDAD/doc/Unidad\%202/2.5.htm\#item0>).

Los ejemplos anteriores son evidencias que hacen plausible afirmar que la proyección metafórica del esquema de imagen equilibrio, y la metáfora conceptual la media aritmética es el equilibrio entre los excesos y los defectos tienen un papel fundamental en la conceptualización de la media aritmética y en la articulación de su complejidad.

\section{Articulación semiótica}

Según Font (2007), hay una relación entre los procesos de representación, metafóricos y los de particularización-generalización, que se puede entender en términos de «aire de familia» (Wittgenstein, 1953), puesto que, de alguna manera, estos procesos hacen intervenir la relación A es B. Si consideramos la estructura A es B como una línea difusa (figura 4), en un extremo podemos situar claramente la relación de subcategorización (particular-general) y, en el otro extremo, la metáfora. La representación, entendida como instrumento de conocimiento, se sitúa en una posición intermedia.

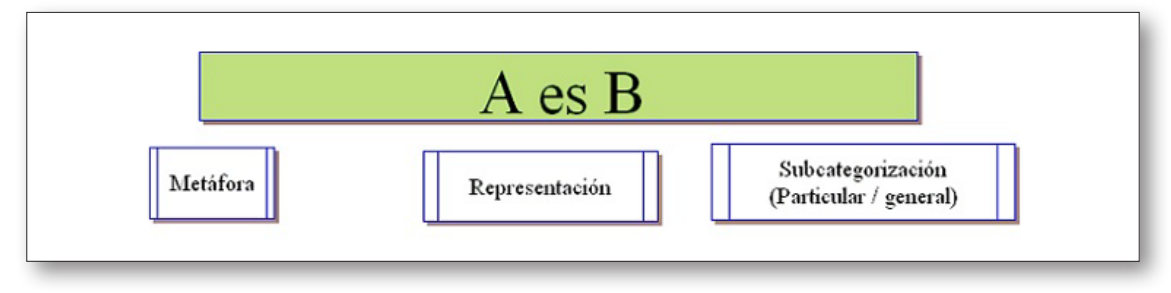

Fig. 4. La estructura A es B (un aire de familia).

Los procesos de esta familia que, según Font (2007), sugieren considerar de alguna manera "A es B», los vamos a llamar en este artículo procesos de articulación semiótica. La representación se puede incluir también en la relación A es B porque, según Peirce, la representación se identifica o se diferencia del objeto representado en función del propósito:

To stand for, that is, to be in such a relation to another that for certain purposes it is treated by some mind as if it were that other. Thus, a spokesman, deputy, attorney, agent, vicar, diagram, symptom, counter, description, concept, premise, testimony, all represent something else, in their several ways, to minds who consider them in that way (Peirce, 1978: \$2273).

Esta articulación la podemos entender como un proceso de encadenamiento semiótico que da como resultado que a una misma expresión se le asignen dos contenidos diferentes que se relacionan entre sí (Rojas, 2012: 52):

Se produce una articulación de sentidos cuando se establece una función semiótica entre dos sentidos diferentes de un mismo objeto matemático primario, esto es, cuando uno de los sentidos (contenido) del objeto primario se convierte en expresión de una nueva función semiótica que tiene como contenido a otro sentido de dicho objeto.

Esta articulación es el resultado de la concatenación de las dos funciones semióticas presentadas. Se tiene un objeto matemático primario al cual se le asignan dos sentidos diferentes. En otras palabras, se produce una articulación de sentidos cuando se establece una nueva función semiótica en la que una de las dos funciones semióticas anteriores juega el papel de expresión. 
Por ejemplo, en la definición que se ha dado anteriormente de valor esperado de una variable aleatoria continua, a la expresión $E(x)=\int_{-\infty}^{+\infty} x f(x) d x$ se le asocia el contenido de ser el resultado de una integral impropia. Si el texto o el profesor relacionan este valor esperado con el de una variable aleatoria discreta y el de esta con la media aritmética, el alumno puede relacionar la integral impropia con la media aritmética, por ejemplo mediante una trama encadenada de funciones semióticas como la siguiente (tabla 3 ).

Tabla 3.

Articulación semiótica mediante una cadena de funciones semióticas

\begin{tabular}{|c|c|c|c|}
\hline \multicolumn{3}{|c|}{ Expresión } & Contenido \\
\hline \multicolumn{2}{|c|}{ Expresión } & Contenido & Media aritmética \\
\hline Expresión & Contenido & $\begin{array}{c}\text { Valor esperado de una } \\
\text { variable aleatoria discreta }\end{array}$ & \\
\cline { 1 - 2 }$E(x)=\int_{-\infty}^{+\infty} x f(x) d x$ & $\begin{array}{c}\text { Valor que resulta de cal- } \\
\text { cular la integral impropia }\end{array}$ & \\
\cline { 1 - 2 } & &
\end{tabular}

Pero si el autor del texto o el profesor no se preocupa de relacionar el valor esperado de una variable aleatoria continua con la media aritmética, el alumno puede considerar que estas dos nociones no se hallan relacionadas entre sí.

La articulación semiótica se puede considerar como el resultado de una trama encadenada de funciones semióticas como las de la figura anterior, o bien como resultado de cadenas semióticas de acuerdo con el modelo triádico de semiosis de Peirce. Por ejemplo, en Presmeg (2006) se usa el modelo de Pierce para reflexionar sobre el papel que juegan las cadenas semióticas con relación al estándar «conexión» del NCTM (2000).

\section{DE UNA VISIÓN COMPLEJA A UNA VISIÓN UNITARIA}

En la primera parte de este artículo, hemos aplicado una visión compleja sobre la noción de media aritmética que nos ha llevado a considerar que esta se presenta en una red de configuraciones epistémicas (figura 3). Desde la perspectiva unitaria, la cuestión que cabe preguntarse es ¿̨cómo se puede considerar que en todas ellas está implicada la noción de media aritmética? A esta pregunta el EOS da una respuesta que se basa en una explicación no realista de cómo los objetos matemáticos emergen de las prácticas matemáticas. Lo que en los planteamientos filosóficos de tipo platonista o empirista se considera un objeto matemático con existencia independiente de las personas (por ejemplo, la media aritmética), en el EOS se explica como un objeto virtual o ficticio que emerge de las distintas maneras de ver, hablar, operar, etcétera globalmente (holísticamente) sobre todos los objetos primarios de la configuración epistémica. Dicho en otros términos, este objeto sería el contenido al que se refiere o indica globalmente, explícita o implícitamente, el par (prácticas matemáticas, configuración epistémica que las activa). Este objeto virtual primero se considera la referencia global de una configuración epistémica y después del conjunto de varias configuraciones epistémicas.

En el EOS (Font, Godino y Gallardo, 2013), se considera que el proceso por el que los objetos matemáticos emergen a partir de las prácticas es complejo y que deben ser distinguidos, al menos, dos niveles. En un primer nivel, emergen representaciones, definiciones, proposiciones, procedimientos, 
problemas y argumentos (objetos primarios) que se organizan en configuraciones epistémicas que permiten realizar prácticas matemáticas en diferentes contextos. En un segundo nivel, emerge una referencia global asociada a una o varias configuraciones epistémicas. La emergencia de este objeto virtual o ficticio se puede explicar por una combinación de efectos de las diferentes dualidades consideradas en el EOS. En este enfoque se considera que los objetos primarios matemáticos que intervienen en las prácticas matemáticas, según el juego de lenguaje en el que participan (Wittgenstein, 1953), pueden ser considerados desde diferentes maneras de "estar participando», las cuales se agrupan en las siguientes facetas o dimensiones duales: unitaria-sistémica, expresión-contenido, ostensivo-no ostensivo, personal-institucional, extensivo-intensivo.

La dualidad unitaria-sistémica permite considerar una configuración epistémica, por ejemplo la del cuadro 1 (CE6), como la descripción de un objeto (media aritmética). Este objeto está definido como $\mathrm{P}=(\mathrm{a}+\mathrm{b}) / 2$ y esta definición forma parte de la configuración epistémica (CE6) en la que hay propiedades que se asume que pertenecen al objeto llamado media aritmética, y procedimientos que se entiende que son aquellos que permiten que el objeto se use. El resultado es que se considera que hay un objeto llamado «media aritmética», que sirve como una referencia global a la configuración epistémica C6. Esta misma dualidad lleva a considerar que el conjunto formado por varias configuraciones (figura 3) es una descripción de un objeto (media aritmética).

Las otras dualidades también son importantes para delimitar propiedades que se le atribuyen al objeto. La dualidad expresión-contenido permite duplicar el objeto considerando la representación y el objeto representado como objetos diferentes (esto lleva a pensar que las diferentes configuraciones son representaciones o descripciones parciales del objeto media aritmética). La dualidad ostensivo-no ostensivo permite considerar que el objeto representado es un objeto ideal diferente de sus representaciones materiales; la dualidad extensivo-intensivo lleva a considerar, normalmente, este objeto como algo general, y la dualidad personal-institucional, que es objetivo. La combinación de estas dualidades produce la emergencia de una referencia global sobre la cual se pueden realizar determinadas acciones. Ahora bien, esta referencia global en la actividad matemática se concreta en una configuración epistémica determinada. Por tanto, lo que se puede hacer con este objeto está determinado por esta configuración.

En el EOS, el objeto que juega el papel de referencia global se puede considerar como único por razones de simplicidad y, a la vez, como múltiple ya que, metafóricamente, se puede decir que estalla en una multiplicidad de objetos primarios agrupados en diversas configuraciones.

En el contexto de la actividad matemática, este objeto virtual o ficticio se presenta en forma de configuración epistémica, y lo que se puede hacer con este objeto está determinado por la configuración en la que este objeto se «presenta». Por otra parte, hay que tener en cuenta que este objeto emerge con el tiempo desde varios sistemas de prácticas matemáticas diferentes. Por esta razón, este objeto puede ser considerado como singular y plural. Singular por razones de simplicidad, y plural porque, en cada subconjunto de prácticas, la configuración de objetos en la que se presenta varía, posibilitando prácticas diferentes (Font, Godino y Gallardo, 2013).

En cierta manera, podemos considerar que este objeto es uno y muchos a la vez. Por una parte, lo podemos considerar de manera unitaria como un emergente de varios sistemas de prácticas diferentes (y de las configuraciones que las activan), en este caso sería el objeto asociado al sistema de pares (prácticas matemáticas, configuraciones que las activan). Esta asociación explicaría que se considere que el objeto se puede definir de diferentes maneras equivalentes, que se puede representar por distintas representaciones, etcétera. Por otra parte, podemos considerar que, en cada configuración, el objeto asociado es diferente.

La visión unitaria de la media aritmética es, según el EOS, el resultado de la combinación de las diferentes dualidades contempladas en este enfoque sobre la red de configuraciones analizadas en la 
figura 3. Ahora bien, en este artículo se muestra que la combinación de estas dualidades para generar la emergencia del objeto media aritmética, como objeto unitario, se basa en la existencia de una especie de eje transversal que articula las diferentes configuraciones (un especie de invariante epistémico) que está relacionado con la propiedad de que los excesos se compensan con los defectos $(\mathrm{P}=\mathrm{a}+\mathrm{E}, \mathrm{P}=$ $\mathrm{b}+\mathrm{D}, \mathrm{E}=-\mathrm{D})$. Este «invariante epistémico» se presenta tanto en formato numérico como gráfico, lo cual se ilustra en la figura 5 .

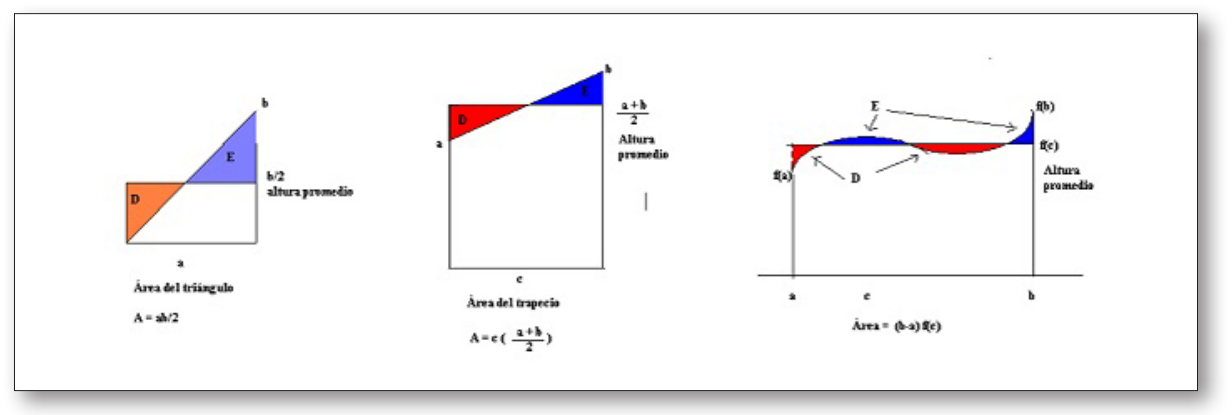

Fig. 5. Registro gráfico del invariante epistémico: los excesos se compensan con los defectos.

\section{CONSIDERACIÓN FINAL}

En este artículo, se ha realizado una reflexión teórica, en el marco del Enfoque ontosemiótico de la cognición e instrucción matemática, que profundiza en los mecanismos de articulación de la complejidad asociada al objeto matemático. Para ello, como contexto de reflexión, se ha utilizado el objeto matemático «media aritmética». A su vez, se presenta una visión integrada de los diferentes tipos de articulación (niveles de generalización, proyecciones metafóricas y tramas de funciones semióticas).

Los principios y estándares del NCTM (2000) consideran el proceso de conexión, que es entendido como aquel que permite conectar diferentes contenidos matemáticos entre sí y también permite conectar las Matemáticas con contextos extramatemáticos. La doble mirada complejidad-articulación aplicada a un objeto matemático (la media aritmética en este caso) permite profundizar en este proceso. Por una parte, la complejidad, estructurada en términos de un conjunto de configuraciones epistémicas, precisa cuáles son los componentes que hay que conectar. Por otra parte, los tres tipos de articulación considerados (generalización, proyección metafórica y articulación semiótica) precisan y concretan la noción de conexión entre estos componentes, en particular la conexión intramatemática.

Introducir la reflexión sobre la dialéctica complejidad-articulación en el proceso de enseñanzaaprendizaje (utilizando las herramientas que se han utilizado en este artículo: configuración epistémica y tipología de procesos de articulación) de un objeto matemático tiene la ventaja de que nos permite generar criterios de calidad matemática de un proceso de enseñanza-aprendizaje para este objeto. Por una parte, la complejidad nos da un marco de referencia para generar el criterio de representatividad (en el sentido de que la secuencia de tareas es una muestra representativa de la complejidad del objeto que se quiere enseñar). A su vez, la tipología de procesos de articulación ayuda a concretar el proceso de conexión, que es uno de los procesos que se tienen en cuenta para hablar de riqueza matemática de una secuencia de tareas (se habla de riqueza en el sentido de que la resolución de las tareas propuestas conlleva la realización de procesos relevantes en la actividad matemática, como son la argumentación, la resolución de problemas, la modelización y, en particular, el proceso de conexión). 


\section{AGRADECIMIENTOS}

Trabajo realizado en el marco del proyecto EDU2012-32644 del Ministerio de Economía y Competitividad de España.

\section{REFERENCIAS}

Acevedo, J. I. (2008). Fenómenos relacionados con el uso de metáforas en el discurso del profesor. El caso de las gráficas de funciones. Tesis doctoral. Universitat de Barcelona.

Arquímedes (1960). Les aeuvers complètes d'Archimède (Tome I). París, France: Vaillant-Carmenne S. A.

Badillo, E. (2003). La Derivada como objeto matemático y como objeto de enseñanza y aprendizaje en profesores de matemática de Colombia. Tesis doctoral. Universitat Autònoma de Barcelona.

Baker, B.; Cooley, L. y Trigueros, M. (2000). A Calculus Graphing Schema. Journal for Research in Mathematics Education, 31 (5), pp. 557-578.

http://dx.doi.org/10.2307/749887

Barceló, R.; Bujosa, J. M.; Cañadilla, J. L.; Fargas, M. y Font, V. (2002). Matemàtiques 1. Barcelona: Castellnou.

Barquero, B.; Bosch, M. y Gascón, J. (2011). Los Recorridos de Estudio e Investigación y la modelización matemática en la enseñanza universitaria de las Ciencias Experimentales. Enseñanza de las Ciencias. 29(3), pp. 339-352.

Barquero, B.; Bosch, M. y Gascón, J. (2013). The ecological dimension in the teaching of modelling at university level. Recherches en Didactique de Mathématiques, 33(3), pp. 307-338.

Batanero, C. (2000). Significado y comprensión de las medidas de posición central. Uno. Revista de Didáctica de las Matemáticas, 25, pp. 41-58.

Callingham, R. (1997). Teachers' multimodal functioning in relation to the concept of average. Mathematics Education Research Journal, 9(2), pp. 205-224.

http://dx.doi.org/10.1007/BF03217311

Chevallard, Y. (1992). Concepts fondamentaux de la didactique: perspectives apportées par une approche anthropologique. Recherches en Didactique des Mathématiques, 12(1), pp. 73-112.

Сobo, B. y Batanero, C. (2004). Significados de la media en los libros de texto de secundaria. Enseñanza de las Ciencias, 22(1), pp. 5-18.

D’Amore, B.; Fandiño, M.; Iori, M. y Matteuzzi, M. (2015). Análisis de los antecedentes histórico-filosóficos de la «paradoja cognitiva de Duval». Revista Latinoamericana de Investigación en Matemática Educativa (en prensa).

Font, V. (2007). Una perspectiva ontosemiótica sobre cuatro instrumentos de conocimiento que comparten un aire de familia: particular-general, representación, metáfora y contexto. Educación Matemática, 19 (2), pp. 95-128.

Font, V.; Godino, J. D. y Gallardo, J. (2013). The emergence of objects from mathematical practices. Educational Studies in Mathematics, 82, pp. 97-124. http://dx.doi.org/10.1007/s10649-012-9411-0

García Cruz, J. A. y Garrett, A. J. (2008). Understanding the Arithmetic Mean: A Study with Secondary and University Students. Journal of the Korea Society of Mathematical Education Series D: Research in Mathematical Education, 12(1), pp. 49-66.

Godino, J. D.; Batanero, C. y Font, V. (2007). The onto-semiotic approach to research in mathematics education. ZDM. The International Journal on Mathematics Education, 39 (1-2), pp. 127-135. http://dx.doi.org/10.1007/s11858-006-0004-1 
Godino, J. D.; Font, V.; Wilhelmi, M. R. y Arrieche, M. (2009). ¿Alguien sabe qué es el número? UNIÓN, Revista Iberoamericana de Educación Matemática, 19, pp. 34-46.

Godino, J. D.; Font, V.; Wilhelmi, M. y Lurduy, O. (2011). Why is the learning of elementary arithmetic concepts difficult? Semiotic tools for understanding the nature of mathematical objects. Educational Studies in Mathematics, 77(2), pp. 247-265.

http://dx.doi.org/10.1007/s10649-010-9278-x

Johnson, M. (1987). The Body in the mind: The bodily basis of meaning, imagination, and reason. Chicago, IL: Chicago University Press.

Lakatos, I. (1981). Matemáticas, ciencia y epistemología. Madrid: Alianza Editorial.

Lakoff, G. y Nuñez, R. (2000). Where mathematics comes from: How the embodied mind brings mathematics into being. New York, NY: Basic Books.

Leavy, A. y O'Loughlin, N. (2006). Preservice teachers understanding of the mean moving beyond the arithmetic average. Journal of Mathematics Teacher Education, 9(1), pp. 53-90.

http://dx.doi.org/10.1007/s10857-006-9003-y

National Council of Teachers of Mathematics (2000). Principles and Standards for School Mathematics. Reston, Virginia: The Council.

Needham, J. (1992). Science and civilisation in china (Vol. 3). Cambridge, UK: Cambridge University Press.

Ortiz, J. J. y Font, V. (2014). Pre-service teachers' common content knowledge regarding the arithmetic mean. REDIMAT, 3(3), pp. 192-219.

Peirce, C. S. (1978). The Collected Papers of Charles Sanders Peirce. Cambridge, MA: The Belknap Press of Harvard University Press.

Piaget, J. y García, R. (1982). Psicogénesis e historia de las ciencias. México D.F.: Editorial Siglo XXI (4. ${ }^{\mathrm{a}}$ edición).

Pino, L.; Godino, J. D. y Font, V. (2011). Faceta epistémica del conocimiento didáctico-matemático sobre la derivada. Educação Matematica Pesquisa, 13(1), pp. 141-178.

Prediger, S.; Bikner-Ahsbahs, A. y Arzarello, F. (2008). Networking strategies and methods for connecting theoretical approaches: First steps towards a conceptual framework. ZDM. The International Journal on Mathematics Education, 40(2), pp. 165-178. http://dx.doi.org/10.1007/s11858-008-0086-z

Presmeg, N. (2006). Semiotics and the «connections» standard: significance of semiotics for teachers of mathematics. Educational Studies in Mathematics, 61(1-2), pp. 163-182. http://dx.doi.org/10.1007/s10649-006-3365-z

RADFORD, L. (2008a). Connecting theories in mathematics education: Challenges and possibilities. ZDM. The International Journal on Mathematics Education, 40(2), pp. 317-327.

RADFORD, L. (2008b). The ethics of being and knowing: Towards a cultural theory of learning. En L. Radford, G. Schubring, y F. Seeger (eds.). Semiotics in mathematics education: Epistemology, history, classroom, and culture. Rotterdam, The Netherlands: Sense Publishers, pp. 215-234.

Rojas, P. J. (2012). Articulación y cambios de sentido en situaciones de tratamiento de representaciones simbólicas de objetos matemáticos. Tesis doctoral no publicada, Bogotà, Colombia, Universidad Distrital Francisco José de Caldas.

Rojas, P. J. (2013). Articulation and Change of Senses Assigned to Representations of Mathematical Objects. Mediterranean Journal for Research in Mathematics Education, 12(1-2), pp. 155-181.

Rojas, P. J. (2015). Objetos matemáticos, representaciones semióticas y sentidos. Enseñanza de las Ciencias, 33(1), pp. 151-165

Rondero, C. (2010). Cálculo promedial. El caso de la media aritmética. Revista Latinoamericana de matemàtica Educativa-RELIME, 13(4-II), pp. 387-408. 
Wilhelmi, M. R.; Godino, J. D. y Lacasta, E. (2007). Configuraciones epistémicas asociadas a la noción de igualdad de números reales. Recherches en Didactique des Mathematiques, 27 (1), pp. 77-120.

Wittgenstein, L. (1953). Philosophical investigations. New York: The MacMillan Company. 


\title{
Articulation of the mathematical complexity of the arithmetic mean
}

\author{
Carlos Rondero \\ Centro de Investigación en Matemáticas. Universidad Autónoma del Estado de Hidalgo \\ rondero@uaeh.reduaeh.mx \\ Vicenç Font \\ Departament de Didàctica de les Ciències Experimentals i de la Matemàtica. Universitat de Barcelona \\ vfont@ub.edu
}

Reflections on the complexity of the mathematical objects and the necessary articulation of the components of such complexity are common in many of the theoretical approaches used in the area of Mathematics Education. In this paper we present a theoretical reflection within the framework of the Onto-Semiotic Approach of the Cognition and Mathematical Instruction, which explores the mechanisms of articulation of the complexity associated to the mathematical object. We present an integrated view of different types of articulation covered in previous research works that have been using this approach as a theoretical framework (layers of generality, metaphoric projection and sequence of semiotic functions). With this purpose, we use the mathematical object «arithmetic mean» as a context of reflection.

We have selected the object «arithmetic mean» because, differing from others such as derivative or integral, which are normally considered as complex, it is usually considered rather as a simple object. However, the arithmetic mean has a mainstreaming characteristic in different areas of mathematics -thus, it turns out to be a good context to reflect about complexity and articulation. The procedure is as follows: 1) we will show the complexity associated to the object arithmetic mean, 2) we will talk about different ways to relate, connect or articulate (in our terminology) the different components of this complexity and, 3) we will reflect on how this articulation allows to switch from a complex view about the arithmetic mean to a unitary vision about such object.

An epistemic configuration is a set of primary objects (language, situation-problem, definitions, procedures, propositions and arguments). For the arithmetic mean, we have been able to identify different intra-mathematical and extra-mathematical contexts, each one of which is associated to a group of mathematical practices and to an epistemic configuration that allow to make them. In the second section, we show the complexity associated to arithmetic mean through a network of seven epistemic configurations. In the third section we consider three mechanisms of articulation of the complexity associated with our context of reflection: the layers of generality, the metaphor and the semiotic articulation, and the way in which its articulation produces the emergence of a unitary object named arithmetic mean, which is considered present within the different configurations of the network. In the fourth section, we reflect on the role played by the articulation in the transition from the complex view to the unitary view of the mathematical object.

We end the article with some final considerations. The unitary vision of the arithmetic mean is, according to the OSA, the result of the combination of the different dualities considered in this approach (expression/content, personal/institutional, ostensive/non-ostensive, extensive/intensive, unitary/systemic) about the network of analyzed epistemic configurations. However, this work shows that the combination of these dualities is based on the existence of some sort of transversal axle which articulates different configurations (epistemic invariant) which is related to the propriety: excesses compensate defects. This «epistemic invariable» is present in numeric format as well as in graphic.

The double view complexity-articulation applied to a mathematical object in this work, the arithmetic mean, in this case, allows furthering in the clarification of the process of connection. The complexity, structured in terms of a group of epistemic configurations, indicates precisely the components to be connected. Separately, the three types of articulation contemplated (generalization, metaphoric projection and semiotic articulation) make the notion of connection precise and concrete. 
